\title{
Avaliação produtiva e sanitária em bovinos Nelore confinados sob metafilaxia antimicrobiana com tildipirosina
}

Layane Queiroz Magalhães ${ }^{[a]}$, Anderson Lopes Baptista ${ }^{[a]}$, Pedro de Almeida Fonseca ${ }^{[a]}$, Guilherme Lobato Menezes ${ }^{[a]}$, Felipe Antunes Magalhães ${ }^{[a]}$, Geison Morel Nogueira ${ }^{[a]}$, Selwyn Arlington Headley ${ }^{[b]}$, João Paulo Elsen Saut ${ }^{[a]}$

\footnotetext{
[a] Laboratório de Saúde em Grandes Animais, Faculdade de Medicina Veterinária, Universidade Federal de Uberlândia (UFU), Uberlândia, MG, Brasil

${ }^{[b]}$ Departamento de Medicina Veterinária Preventiva, Universidade Estadual de Londrina (UEL), Londrina, PR, Brasil
}

*Autor correspondente

e-mail: layanequeirozmagalhaes@gmail.com

\section{Resumo}

A metafilaxia objetiva o controle da doença respiratória (DRB) aguda e deve ser implantada em populações que tenham histórico de DRB. Com o uso destes protocolos há redução da doença individual e da incidência no rebanho. A hipótese foi de que a metafilaxia, na entrada do confinamento, reduz os índices de morbidade e mortalidade em animais com alto risco de DRB e melhora índices produtivos. Com o objetivo de avaliar a eficácia do tratamento metafilático, com $4 \mathrm{mg} / \mathrm{Kg}$ de tildipirosina, na ocorrência de DRB e no desempenho produtivo, quando realizado apenas em metade dos bovinos em lotes com alto risco de DRB, foram realizados dois experimentos utilizando bovinos machos, não castrados, Nelore, idade (18 - 24 meses), peso de entrada $(366,9 \pm 19,9 \mathrm{Kg})$, deslocamento prévio $(657,9 \pm 302,3 \mathrm{Km})$ e período de descanso $(10,4 \pm 15,6$ dias). No experimento $1(n=1.780)$, os animais foram divididos em dois grupos: a) grupo controle $(n=628)$, bovinos sem metafilaxia e separados em seis lotes; b) grupo metafilaxia ( $n=1.152)$, metade dos bovinos foram tratados com tildipirosina na dose única de $4 \mathrm{mg} / \mathrm{Kg}$ PV, SC ( $\mathrm{n}=584)$ e a outra solução salina ( $\mathrm{NaCl}$ $0,9 \%)$ na dose de $0,02 \mathrm{~mL} / \mathrm{Kg}$ PV, SC $(\mathrm{n}=568)$ e alocados em dez lotes. No experimento $2(\mathrm{n}=1152)$, foram utilizados os animais do grupo metafilaxia pertencentes ao experimento 1 . Os animais foram denominados tratados ( $\mathrm{n}=584 ; 4 \mathrm{mg} / \mathrm{Kg}$ tildipirosina, SC) e não tratados ( $\mathrm{n}=568 ; 0,02 \mathrm{~mL} / \mathrm{Kg}$ Solução salina 0,9\%, SC). A morbidade por DRB foi de 4,77\% (85/1780) e não houve nenhuma morte por DRB. Não foi observada diferença $(P=0,02)$ na morbidade entre os animais do grupo controle $(6,5 \%$ - 41/628) e grupo metafilaxia $50 \%$ (3,8\% - 44/1152). Dentre índices produtivos, o GDC (P = 0,002) e o GPD (P = 0,039) foram maiores nos animais do grupo metafilaxia. Não houve diferença $(\mathrm{P}=0,691)$ na morbidade entre os animais tratados $\mathrm{e}$ não tratados no experimento 2: 3,1\% (18/584) e 4,6\% (26/568), respectivamente. 0 peso final $(\mathrm{P}=0,058) \mathrm{e}$ 
GPD $(\mathrm{P}=0,056)$ do grupo não tratados apresentaram tendência em ser menores do que os do grupo tratados $(535,40 \pm 34,20 \mathrm{Kg})$. Conclui-se que a metafilaxia com tildipirosina, realizada em metade de animais com alto risco para doenças respiratórias na entrada de confinamentos, reduz a morbidade da DRB e melhora o desempenho produtivo. 